\title{
Rare Coexistance of Ileal Diverticulosis, Crohn's Disease and Small Bowel Adenocarcinoma: Report of a Case
}

\author{
ELEFTHERIOS SPARTALIS ${ }^{1}$, NIKOLAOS GARMPIS ${ }^{1}$, MICHAEL SPARTALIS ${ }^{1}$, CHRISTOS DAMASKOS ${ }^{1}$, \\ DEMETRIOS MORIS ${ }^{2}$, ANTONIOS ATHANASIOU ${ }^{3}$, PARASKEVAS GKOLFAKIS ${ }^{4}$, \\ PENELOPE KORKOLOPOULOU $^{5}$, DIMITRIOS DIMITROULIS ${ }^{1}$ and DIMITRIOS MANTAS ${ }^{1}$ \\ ${ }^{1}$ Second Department of Propaedeutic Surgery, Medical School, \\ "Laikon" General Hospital, University of Athens, Athens, Greece; \\ ${ }^{2}$ Department of Surgery, The Ohio State University Comprehensive Cancer Center, \\ The Ohio State University, Columbus, OH, U.S.A.; \\ ${ }^{3}$ Department of Surgery, Queen Elizabeth Hospital, \\ University Hospitals Birmingham NHS Foundation Trust, Birmingham, U.K.; \\ ${ }^{4}$ Hepatogastroenterology Unit, Medical School, "Attikon" General Hospital, University of Athens, Athens, Greece; \\ ${ }^{5}$ Department of Pathology, University of Athens Medical School, Athens, Greece
}

\begin{abstract}
Background/Aim: Adenocarcinoma is one of the most common malignant tumors of the small intestine complicating Crohn's disease. However, the coexistence of both conditions with diverticulosis of small bowel in young age makes this coincidence rare and clinical diagnosis very difficult. Case Report: We report a case of a woman admitted to our Department with acute abdominal pain and fever. The surgical and histological investigation, revealed a rare coexistence that has never been mentioned in the published medical literature. Conclusion: Ileal diverticulosis is not frequent and often asymptomatic as well as adenocarcinoma of the small bowel. In this case, those diseases along with Crohn's disease led the patient to acute symptoms.

It has been recognized that patients with Crohn's disease (CD) are at increased risk of developing malignant lesions (1). Small bowel adenocarcinoma develops in $1.5 \%$ of patients who have longstanding $\mathrm{CD}$ and is very rarely diagnosed preoperatively because of its rarity, overlapping imaging features and lack of reported cases $(1,2)$. Adenocarcinoma in CD is more often poorly differentiated and has a worse prognosis than primary adenocarcinoma of
\end{abstract}

This article is freely accessible online.

Correspondence to: Dr. Eleftherios Spartalis, Vasilissis Sofias 49, Athens 106 76, Greece. Tel: +30 6974714078, e-mail: eleftherios.spartalis@gmail.com

Key Words: Ileal diverticulosis, Crohn's disease, small bowel adenocarcinoma. small bowel. The median age at diagnosis is 49 years (2). The first reports of CD patients who subsequently developed cancer of the large or small intestine were published in 1948 and 1956, subsequently (3). In 1746, Hamburger made the first case report of duodenal carcinoma and the first review of malignant small bowel neoplasms was published in 1876 by Leichtenstein $(2,3)$.

Prevalence of small intestinal diverticula on autopsy ranges from $0.06 \%$ to $1.3 \%$ (4). The prevalence increases with age, peaking at the sixth and seventh decade. Eighty percent of diverticula occur in the jejunum (JID), $15 \%$ in the ileum and 5\% in both (4). If conservative management is unresponsive, successful treatment largely depends on surgical resection with primary anastomosis (5). Small bowel diverticula were first described by Soemmering and Baille in 1794 (6). The first operation was performed by Gordinier and Sampson in 1906 on a patient with partial small bowel obstruction, due to inflamed JID; resection of the involved jejunal segment was curative $(6,7)$.

We present, to the best of our knowledge, the very first case of concomitant adenocarcinoma of small bowel and JID in a patient with $C D$ presenting with acute abdominal pain.

\section{Case Presentation}

A 38-year-old Caucasian female presented to the emergency room due to abdominal pain and intense diarrhea syndrome for $48 \mathrm{~h}$. She also complained for sickness, anorexia and fever (her temperature was $39^{\circ} \mathrm{C}$ ) with chills. The use of painkillers had no effect on the pain. There was no vomiting nor nausea but in the last $24 \mathrm{~h}$ she complained for urinary retention. The patient had diagnosed with Erythema 
nodosum two years ago without taking any medication for that. The existence of inflammatory bowel disease (IBD) has never been evaluated before. There was no history of previous abdominal surgeries.

Physical examination revealed dehydration, diffuse abdominal tenderness more severe at the left lower quadrant, with rebound pain. The pregnancy test was negative and there was a diffuse sensitivity in gynecological examination. A plain radiograph and ultrasound of the abdomen at the emergency were unremarkable. Computer tomography (CT) of the abdomen revealed thickening of the terminal ileum, increasing dimensions and thickening of the cecum. Additionally, an endopelvic abscess $(40 \mathrm{ml})$ behind the sigmoid was also found (Figure 1).

An exploratory laparotomy with midline incision was made, revealing diverticulosis of small intestine, distended cecum, Meckel diverticulum and pus in the peritoneal cavity. A part of terminal ileum with the anterior wall of sigmoid was coalesced in pelvis. Resection of the terminal ileum and abscess drainage were performed. Reconstruction of the intestinal transit was achieved with an end-to-end ileocolic anastomosis.

Histologically (Figures 2-6), the resected surgical specimen of final ileum $(24.5 \mathrm{~cm})$ was characterized by multiple inflamed pseudodiverticula, ulcers and focused pylorus-like metaplasia with eosinophil and neutrophil cells. Moreover, epithelium-type granulomas and inflammation involving all layers of the intestinal wall, with abscesses and lymph nodes, were also found, setting the diagnostic direction to CD. Finally, adenocarcinoma with $>50 \%$ mucus was detected by well-differentiated neoplastic cells and Paneth cells among them. The malignancy infiltrated the intestinal wall, disintegrating the muscularis coat without extension to serosal layer. All lymph nodes (17 resected) were negative for malignant cells (T3NOMx). The specimen of Meckel was characterized by heterotopic gastric mucosa with focused adenoma of low grade dysplasia.

The patient was discharged in good general condition with the guidance for oncological consultation. However, one week after her discharge, the patient was admitted again due to abdominal pain, fever, chill and in-appetence. She had suffered from constant pain located in sub-abdomen for 2 days, but denied diarrhea and nausea. Ultrasound of abdomen showed abscess of $10 \mathrm{~cm}$ in Douglas foramen and small pelvis. Abscess drainage and wide-spectrum antibiotics were administered. Oncological follow-up with CT-scan and clinical re-evaluations in the first 6 and 12 months revealed no disease recurrence.

\section{Consent}

Written informed consent was obtained from the patient for publication of this case report and accompanying images.
A copy of the written consent is available for review by the Editor-in-Chief of this journal.

\section{Discussion}

The small bowel corresponds to $75 \%$ of the digestive tract length and $90 \%$ of its mucosal surface area, but surprisingly the frequency of neoplasms is lower than in the stomach or the large bowel. This apparent "neoplastic resistance" finds its eventual explanation in the low bacterial content capable of transforming food components into carcinogens, along with the large amount of alkaline fluid secretion, the rapid transit time, and the high concentration of the enzyme benzopyrene hydroxylase, which reduce the mucosal contact with potential carcinogens (8). The mean age of diagnosis of small bowel tumors is 60 years of age, with a slight male preponderance. Adenocarcinoma is the most common histologic type (30-50\%) (7).

About $2 \%$ of patients affected by CD will develop cancer in the course of their disease, and, in contrast to the patients with ulcerative colitis (UC), those with CD are at risk for developing malignancy even in the first decade of their disease (9). The coexistence of CD with adenocarcinoma is predominantly seen in men, in the patients with excluded loop and most frequently in the distal ileum in an area of active disease (9). Most patients presenting with adenocarcinoma complicating CD have a high-grade malignancy with lymph node involvement or distant metastases, because the similarity of the presenting symptoms and of the radiography of these pathologies creates diagnostic problems for the physician and make an early diagnosis impossible (1). The most frequent presenting symptoms are abdominal pain, anemia, and small bowel obstruction. In most cases $(88 \%)$ adenocarcinoma is found incidentally at surgery (2).

JID is a rare disorder and the course of the disease is mostly asymptomatic. Its reported incidence varies from $0.05 \%$ to $6 \%$ (4). This rare entity should not be regarded as a clinically insignificant finding. It may be difficult to make a preoperative diagnosis. Diagnosis is often delayed, resulting in unnecessary morbidity and mortality (10). They are most common in the duodenum with a frequency of approximately $5 \%$. They are less common in the ileum (11).

Presenting complaints such as intermittent abdominal pain, constipation and diarrhea, akin to those seen in irritable bowel syndrome, have been demonstrated in up to $90 \%$ of the patients (5). There are no pathognomonic signs or symptoms of small-bowel diverticulitis. However, they may be associated with obscure gastrointestinal bleeding or bacterial overgrowth and may on occasion become impacted with ingested food, become inflamed and present with acute abdominal pain (10).

Complications due to JID include pseudoobstruction, blind loop syndrome, jejuna dyskinesia and chronic diverticulitis 


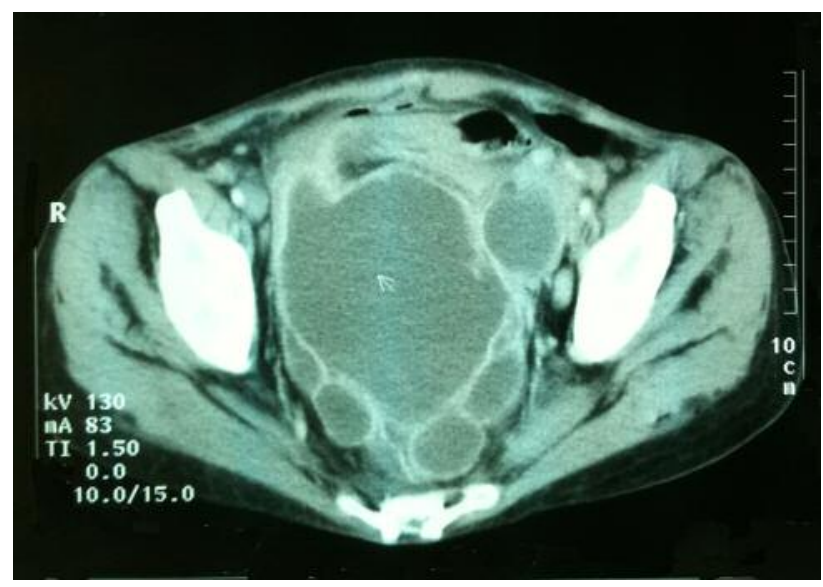

Figure 1. Computer tomography $(C T)$ of the abdomen revealed an endopelvic abscess.

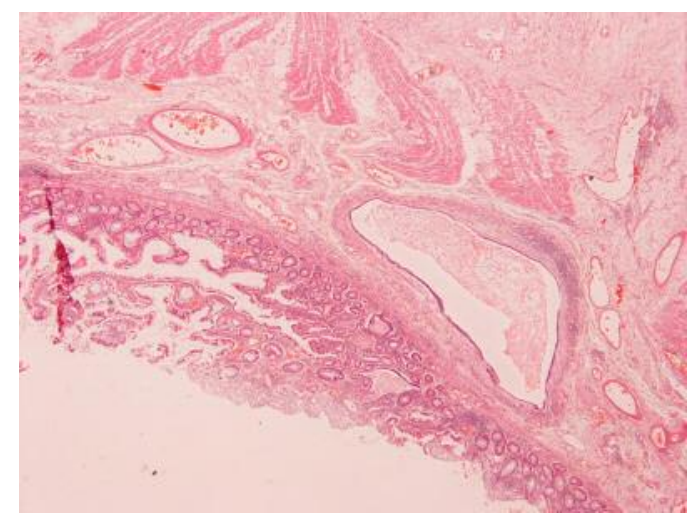

Figure 2. Submucosal neoplastic gland lined by well-differentiated malignant cells.

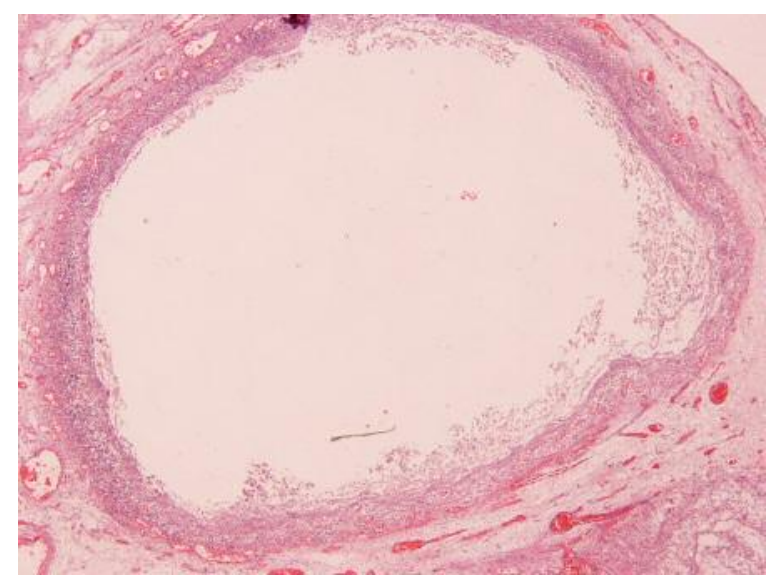

Figure 3. Large abscess formation in submucosal mesenteric fat.

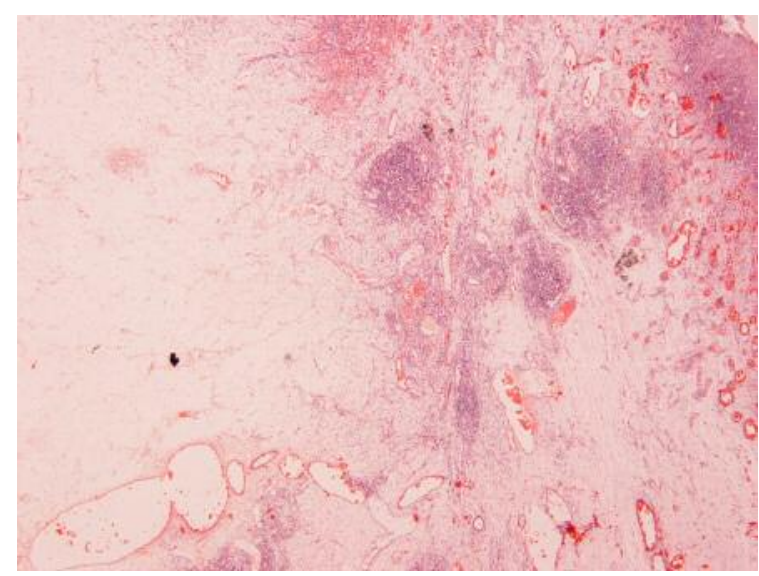

Figure 4. Numerous prominent lymphoid aggregates into the perienteric fat tissue.

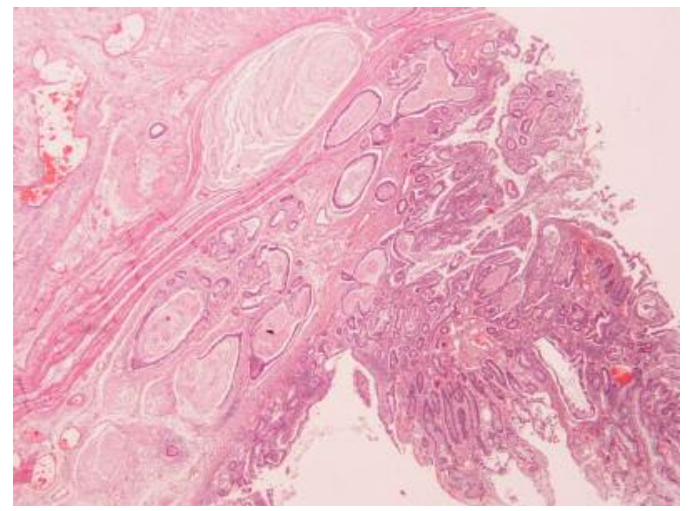

Figure 5. Low-power magnification demonstrating the presence of neoplastic glands filled with mucin, invading the muscularis propria.

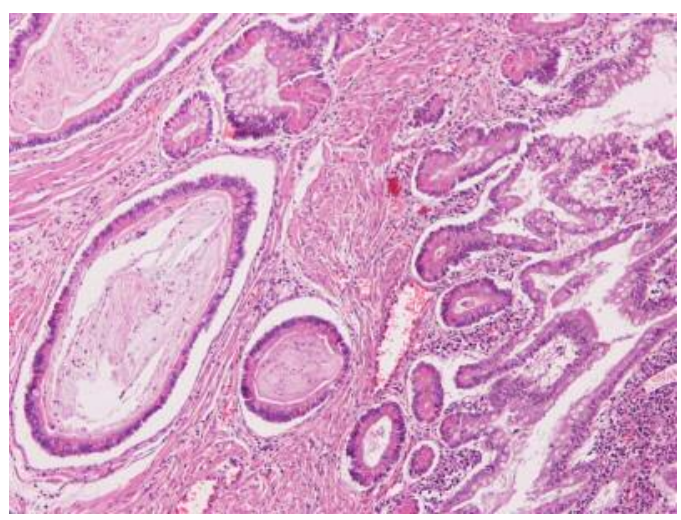

Figure 6. Higher-power magnification demonstrates the welldifferentiated malignant epithelial cells, with Paneth cells among them. The mucin is intraluminal as long as intracellular. 
complicated by the formation of enterolith. More acute complications include perforation, peritonitis, bleeding and fistula formation (12). The differential diagnosis includes perforated neoplasm, foreign body perforation, small bowel ulceration from nonsteroidal anti-inflammatory drug use, CD and diverticulitis. Perforated neoplasms can be difficult to distinguish from JID. The most likely neoplasm to perforate would be lymphoma.

Resection of the involved area with primary jejunojejunal anastomosis is the surgical management of choice in the presence of perforated JID, hemorrhage, or abscess formation after a failure of a short course of bowel rest and antibiotics (11).

Small bowel diverticula are thin-walled sacculations that, in contrast to Meckel diverticula (13), consist only of mucosa, submucosa, and occasionally a thin layer of serosa without muscle (can thus be referred to as "pseudodiverticula"). They are almost always located on the mesenteric border, at the site of entry of the vasa recta which consist the locus minoris resistentiae of the small bowel (14). This proximity of the fundus of the diverticula to branches of the mesenteric vessels is responsible for the hemorrhagic complications of the disease (15).

The clear predominance of diverticula in the jejunum is probably attributed to the greater diameter of the penetrating vessels in the proximal part of the small bowel. Actually, the number of JID decreases as we move distally from the ligament of Treitz with nearly $80 \%$ of them occurring in the jejunum, $15 \%$ in the ileum, and $5 \%$ in both (4). Their size varies considerably, often reaching a diameter of more than $5 \mathrm{~cm}$. Although it has been suggested that some JID may be congenital (solitary diverticula in young patients may represent partial reduplication of the small bowel), it is now widely accepted that most of these lesions are acquired. The pathogenesis seems to be multifactorial (16).

Regarding the etiology of JID, the current hypothesis focuses on abnormalities in the smooth muscle or myenteric plexus (17). Careful microscopic evaluation of jejunal specimens with diverticula has shown that these abnormalities are of three types: fibrosis and decreased numbers of normal muscle cells, consistent with progressive systemic sclerosis; fibrosis and degenerated smooth muscle cells, suggestive of a visceral myopathy; neuronal and axonal degeneration indicative of visceral neuropathy (17). Any of these abnormalities could lead to distorted smooth muscle contractions of the affected small bowel generating increased intraluminal pressure. The result is herniation of mucosa and submucosa through the weakest mesenteric site of the bowel wall with penetration induced by paired blood vessels from the mesentery (18).

Diverticula are depicted, either on plain films or on CT scans, as sacculations with retained contrast medium after the main bowel lumen has become empty. Enteroclysis, additional diagnostic media, such as ultrasound, computed tomography, endoscopy, capsule endoscopy, intraoperative endoscopy, deep enteroscopy with single or double-balloon and spiral enteroscopy can be used $(19,20)$. Laparoscopy, radiotagged erythrocyte bleeding scans and selective mesenteric arteriography can be performed as well. Laparotomy remains the gold standard for definite diagnosis of asymptomatic and complicated diverticula (16).

Compared with duodenal diverticula, JID are nearly 4 times more likely to develop complications, and nearly 18 times more likely to perforate and develop abscesses (5).

Both CD and JID are seen with sufficient frequency that unless one disease protects from the other, one would occasionally expect to see coincident disease $(21,22)$.

\section{Conclusion}

Perforation of a JID has to be considered in the differential diagnosis of acute abdomen. Although JID with its complications, such as perforation, is difficult to suspect in patients with peritonitis, it should be considered as a possible source of abdominal infection in elderly patients when more common diagnoses have been excluded or in patients with pathophysiological background making them susceptible to JID development, such as CD. Small bowel adenocarcinomas are rare tumors, but their incidence is increasing. Despite being most often sporadic, some predisposing diseases have been identified, among which CD and genetic syndromes. Early diagnosis of small bowel adenocarcinoma remains difficult despite significant radiological and endoscopic progress. We present, to our knowledge, the very first case of concomitant adenocarcinoma of small bowel and JID in a patient with $\mathrm{CD}$ presenting with acute abdominal pain.

\section{Conflicts of Interest}

The Authors declare that they have no competing interests.

\section{References}

1 Cioffi U, De Simone M, Ferrero S, Ciulla MM, Lemos A and Avesani EC: Synchronous adenocarcinoma and carcinoid tumor of the terminal ileum in a Crohn's disease patient. BMC Cancer 5: 157,2005

2 Tirkes AT and Duerinckx AJ: Adenocarcinoma of the ileum in Crohn disease. Abdominal Imag 30(6): 671-673, 2005.

3 Ying LT, Hurlbut DJ, Depew WT, Boag AH and Taguchi K: Primary adenocarcinoma in an enterocutaneous fistula associated with Crohn's disease. Canad J Gastroenterol 12(4): 265-269, 1998.

4 Staszewicz W, Christodoulou M, Proietti S and Demartines N: Acute ulcerative jejunal diverticulitis: case report of an uncommon entity. World J Gastroenterol 14(40): 6265-6267, 2008.

5 Nejmeddine A, Bassem A, Mohamed H, Hazem BA, Ramez B and Issam BM: Complicated jejunal diverticulosis: A case report with literature review. N Am J Med Sci 1(4): 196-199, 2009. 
6 Chugay P, Choi J and Dong XD: Jejunal diverticular disease complicated by enteroliths: Report of two different presentations. World J Gastrointest Surg 2(1): 26-29, 2010.

7 Palascak-Juif V, Bouvier AM, Cosnes J, Flourie B, Bouche O, Cadiot G, Lémann M, Bonaz B, Denet C, Marteau P, Gambiez L, Beaugerie L, Faivre $\mathrm{J}$ and Carbonnel F: Small bowel adenocarcinoma in patients with Crohn's disease compared with small bowel adenocarcinoma de novo. Inflamm Bowel Dis 11(9): 828-832, 2005.

8 Shenoy S: Primary small-bowel malignancy: update in tumor biology, markers, and management strategies. J Gastrointest Cancer 45(4): 421-430, 2014.

9 Aparicio T, Zaanan A, Svrcek M, Laurent-Puig P, Carrere N, Manfredi S, Locher $\mathrm{C}$ and Afchain P: Small bowel adenocarcinoma: epidemiology, risk factors, diagnosis and treatment. Dig Liver Dis 46(2): 97-104, 2014.

10 Moris D and Vernadakis S: Jejunal diverticulosis as the obscure cause of acute abdominal pain. Surgery 155(6): 1093-1094, 2014

11 De Peuter B, Box I, Vanheste R and Dymarkowski S: Smallbowel diverticulosis: imaging findings and review of three cases Gastroenterol Res Pract 2009: 549853, 2009.

12 Patel VA, Jefferis H, Spiegelberg B, Iqbal Q, Prabhudesai A and Harris S: Jejunal diverticulosis is not always a silent spectator: a report of 4 cases and review of the literature. World $\mathrm{J}$ Gastroenterol 14(38): 5916-5919, 2008.

13 Uppal K, Tubbs RS, Matusz P, Shaffer K and Loukas M. Meckel's diverticulum: a review. Clin Anat 24(4): 416-422, 2011.

14 Thomas RA, Gibson S, Paul M and McDonald SW: Jejunal diverticulum with ectopic pancreatic mucosa: was it really a Meckel's diverticulum? Clinical Anat 25(4): 509-512, 2012.
15 Shetty S, Balasundaram P, Muthuraman M and Leelakrishnan V: Jejunal diverticulosis - rare cause of gastrointestinal bleed. Indian J Surg 76(1): 15-16, 2014.

16 Makris K, Tsiotos GG, Stafyla V and Sakorafas GH: Small intestinal nonmeckelian diverticulosis. J Clin Gastroenterol 43(3): 201-207, 2009.

17 Voiosu AM, Patrascu T, Bobirca F and Voiosu TA: Massive diverticulosis of the small intestine. Dig Liver Dis 46(4): 383, 2014.

18 Chiu EJ, Shyr YM, Su CH, Wu CW and Lui WY: Diverticular disease of the small bowel. Hepato-Gastroenterol 47(31): 181184, 2000.

19 Grana L, Pedraja I, Mendez R and Rodriguez R: Jejuno-ileal diverticulitis with localized perforation: CT and US findings. Eur J Radiol 71(2): 318-323, 2009.

20 Chen TH, Chiu CT, Lin WP, Su MY, Hsu CM and Chen PC: Application of double-balloon enteroscopy in jejunal diverticular bleeding. World J Gastroenterol 16(44): 5616-5620, 2010.

21 Gledhill A and Dixon MF: Crohn's-like reaction in diverticular disease. Gut 42(3): 392-395, 1998.

22 Peppercorn MA: The overlap of inflammatory bowel disease and diverticular disease. J Clin Gastroenterol 38(5 Suppl 1): S8-10, 2004.
Received October 17, 2017

Revised November 8, 2017

Accepted November 9, 2017 